\title{
THE NEUROIMAGE VALUE FOR URGENT PSYCHIATRY
}

\author{
C. Mendes 1 , G. Bastos², J. Fonseca² \\ 1 Unidade Local de Saúde do Alto Minho, Psychiatry, Viana do Castelo, Portugal \\ ${ }^{2}$ Centro Hospitalar Univeristário S. João, Neurorradiology, Porto, Portugal
}

\begin{abstract}
Introduction
The purpose of this study was to understand the brain imaging need for psychiatric patients evaluation in an emergency context, studying the findings and their guidance after discharge. The exclusion of organicity is mandatory in many psychiatric diagnosis investigations, in order to confirm that the patient clinical case is not a result of any medical reason. Cases in which a head computed tomography (CT) scan or magnetic resonance imaging (MRI) of the brain are most necessary are: behaviour changes, early psychotic symptoms, manic episodes with psychotic symptoms, and progressive dementia syndromes. These cases, at the presentation, may hide any medical condition and, apart from the brain anatomy study, it is also important to ask for a blood and urine test analysis including toxicological tests.
\end{abstract}

\section{Material and methods}

We analysed brain CT imaging requests by Psychiatric Physicians at the Metropolitan Psychiatric Emergency Department of Porto (UMPP) in the year 2017 (from January to December 2017), guided by them until discharge. We included patients living in the S. João Hospital residential area (Maia, Valongo, Campanhã, Bonfim and Paranhos), where UMPP is located - because were the only whom we had information about after discharge. We had a population of 150 people. The analysis included the study of the clinical presentation which motivated the exam, the image findings and what consequence those findings had on physician practice and patients guidance.

\section{Results}

During one year, at the UMPP, 150 patients had a brain image result and were headed by the Psychiatric Physician until discharge. Some, because of no positive finding (concluding the psychiatric etiology, also after no medical condition explained by analytic findings) were inpatient or outpatient oriented, depending on the severity $(\mathrm{N}=113)$. Others, with some image findings, even if minor, were simply disregarded and assumed the psychiatric condition $(\mathrm{N}=37)$.

At the moment of the discharge, from the 37 patients with some finding in the brain CT-scan, $12(32,4 \%)$ were referred to a Neurology/Internal Medicine outpatient attendance (because of the clinical condition, not mentioning the CT-scan finding), 17 were referred to a Psychiatry outpatient attendance $(45,9 \%)$ and $21,6 \%$ were left with no guidance.

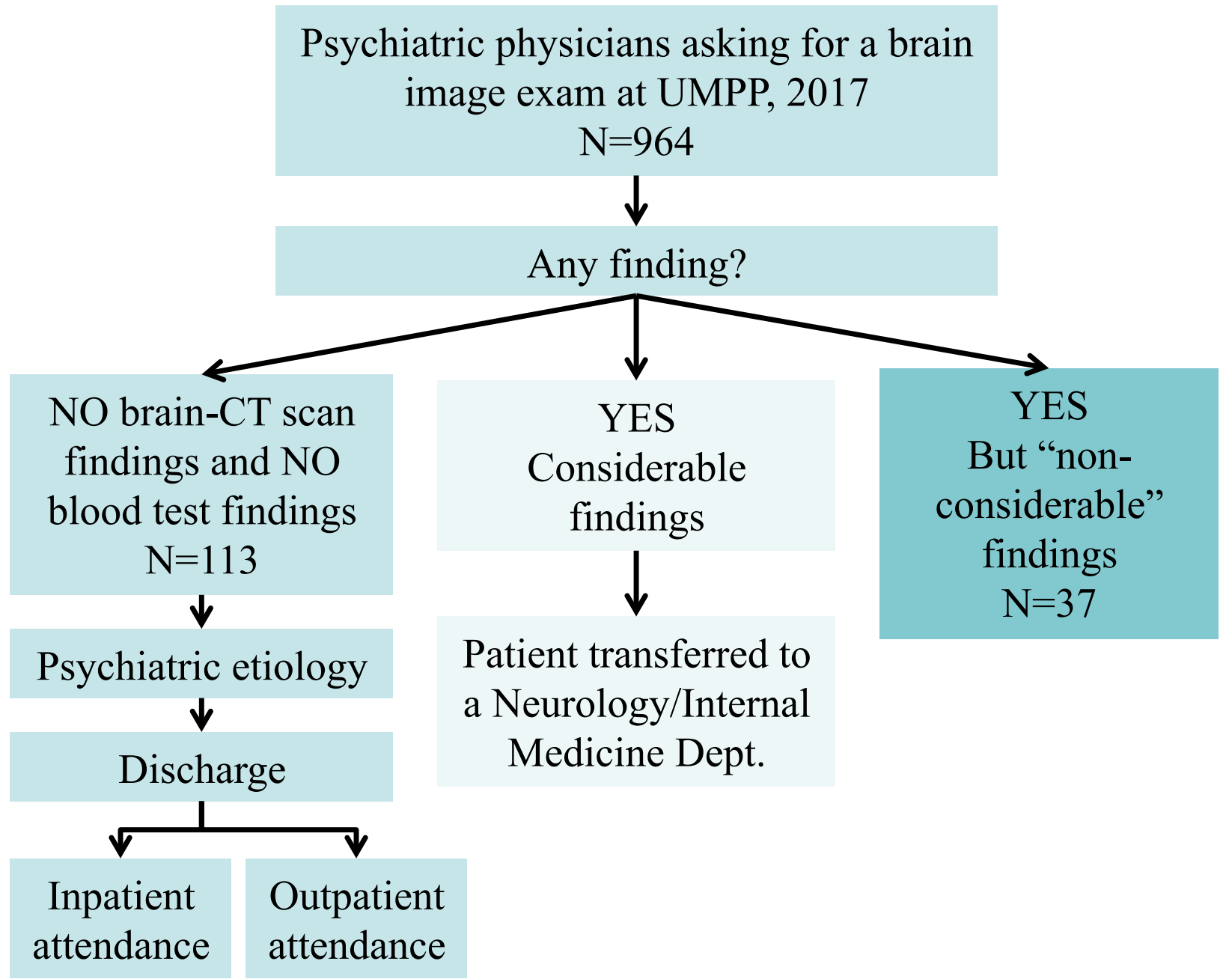

\begin{tabular}{|l|l|}
\hline Brain CT-scan findings & Total \\
\hline Cystic sellar lesion & 3 \\
\hline Atrophy & 9 \\
\hline Enlarged perivascular spaces & 1 \\
Great vessel ischemic chronic lesions & 1 \\
\hline Small vessels ischemic lesions & 18 \\
Small and great vessels ischemic lesions & 1 \\
\hline Arachnoid cystic lesion & 1 \\
Anatomical variation of the ventricular system & 3 \\
\hline Total & 37
\end{tabular}

\section{Discussion and conclusions}

When a Psychiatric Physician asks for a brain image exam, in an emergency department context, looking for any finding which can justify the symptoms, she/he needs to consider any change. For medical and legal reasons, all of the analytic or imaging findings should be correlated and the patient should be transferred to a Neurology or an Internal Medicine Department. From the 150 patients who were discharged by the Psychiatry department, with no other colleague observation, we expected no brain CT scan, physical exam or blood and urine test findings, assuming a psychiatric illness. However, for those 37 patients, brain image was useless, as their findings were ignored. This study may remind us how important are relationships between physicians in an emergency department and how easily they let important information run away from the multidisciplinary discussions and colleague collaborations. 